\title{
Inpatient cost for hip fracture patients managed with an orthogeriatric care model in Singapore
}

\author{
Lester Teong Jin $\underline{T a n}^{1}$, MBBS, MRCs, Seng Joung Wong ${ }^{2}$, Ernest Beng Kee Kwek ${ }^{1}$, MBBS, FRCS
}

INTRODUCTION The estimated incidence of hip fractures worldwide was 1.26 million in 1990 and is expected to double to 2.6 million by 2025. The cost of care for hip fracture patients is a significant economic burden. This study aimed to look at the inpatient cost of hip fractures among elderly patients placed under a mature orthogeriatric co-managed system. METHODS This study was a retrospective analysis of 244 patients who were admitted to the Department of Orthopaedics of Tan Tock Seng Hospital, Singapore, in 2011 for hip fractures under a mature orthogeriatric hip fracture care path. Information regarding costs, surgical procedures performed and patient demographics was collected.

RESULTS The mean cost of hospitalisation was SGD 13,313.81. The mean cost was significantly higher for the patients who were managed surgically than for the patients who were managed non-surgically (SGD 14,815.70 vs. SGD 9,011.38; $p<0.01)$. Regardless of whether surgery was performed, the presence of complications resulted in a higher average cost (SGD 2,689.99 more than if there were no complications; $p=0.011$ ). Every additional day from admission to time of surgery resulted in an increased cost of SGD 575.89, and the difference between the average cost of surgery within 48 hours and that of surgery $>48$ hours was SGD 2,716.63.

CONCLUSION Reducing the time to surgery and preventing pre- and postoperative complications can help reduce overall costs. A standardised care path that empowers allied health professionals can help to reduce perioperative complications, and a combined orthogeriatric care service can facilitate prompt surgical treatment.

Keywords: economic costs, hip fractures, orthogeriatrics

\section{INTRODUCTION}

The estimated incidence of hip fractures was 1.26 million in 1990 and is expected to double to 2.6 million by 2025 . $^{(1)}$ These estimates are from a study conducted by Gullberg et al on the incidence of hip fractures and population trends. The authors projected that the estimated incidence of hip fractures would increase to 4.5 million by the year 2050. They predicted that the majority of this increase would occur in Asia, with $37 \%$ of all hip fractures occurring in Asia in 2025 compared to $26 \%$ in 1990. ${ }^{(1)}$ With the higher incidence of hip fractures comes a significant economic burden; the cost of care for hip fracture patients ranges from CAD 650 million a year in Canada ${ }^{(2)}$ to over GBP 2 billion a year in the United Kingdom. ${ }^{(3)}$ Hip fractures that are treated using a standardised hip fracture protocol and co-managed by orthopaedic surgeons and geriatricians have been shown to result in significantly lower hospitalisation costs, ${ }^{(4)}$ as well as lower mortality rates, shorter length of hospital stays and reduced time-to-surgery.

The average lifespan of Singaporeans has increased. In 2000, it was 76.0 years for males and 80.0 years for females, while in 2014, it was 80.5 years for males and 84.9 years for females. ${ }^{(5)}$ The percentage of the population aged above 65 years has also increased, from $7.4 \%$ in 2003 to $9.9 \%$ in $2012 .{ }^{(5)}$ With the increasing life expectancy and ageing population, the incidence of osteoporotic hip fractures will likely continue to increase; hence, hip fractures will likely continue to be a challenge for the Singapore healthcare system.

The present study aimed to examine the inpatient cost of hip fractures among elderly patients placed under a mature orthogeriatric co-managed system. Previously published data from Singapore showed that the cost of a hip fracture in 1993 and 2001 was SGD 7,367 and SGD 10,515, respectively. ${ }^{(6,7)}$ We aimed to provide an update on that cost and to evaluate the factors associated with increased costs. We hypothesised that delays to surgery would not only result in increased morbidity, but also an associated increase in the financial cost of a hip fracture.

\section{METHODS}

All patients aged $>60$ years who were admitted to the Department of Orthopaedics, Tan Tock Seng Hospital, Singapore, for hip fractures from January-June 2011 were identified. The case notes of these patients were retrospectively reviewed. Patients who were aged $>60$ years and had primary fractures of the hip, either intertrochanteric or neck of femur fractures (i.e. AO 31-A or AO 31-B fractures), were included in the study. Patients were excluded if they had femoral shaft fractures; died before completion of the treatment; were discharged to other hospitals during the course of treatment; were discharged against medical advice; had complications of previous total hip replacement; or had pathological, periprosthetic, subtrochanteric or isolated greater trochanter fractures.

The included patients were treated either surgically or non-surgically, depending on the outcome of a review with an orthopaedic consultant and a geriatrician, and a discussion between the attending doctor and the patient and his/her family. All intertrochanteric fractures were treated with surgical fixation, while femoral neck fractures were treated with either total hip arthroplasty or bipolar hemiarthroplasty. No randomisation was 
involved, as treatment was administered based on the patient's fracture type.

The patients' case notes and final hospitalisation bills were reviewed, and relevant data was collected and analysed. Data collected included patient demographics (e.g. age, gender and comorbidities), length of hospital stay, type of fracture, type of treatment (surgical or non-surgical), time from admission to surgery and complications during the hospital stay. In addition, data on the overall cost of the inpatient stay, the cost difference between cases managed surgically and those managed non-surgically, implant costs, and the costs incurred due to complications was collected.

The overall average cost incurred by all the patients and the average costs incurred by the surgical and non-surgical groups were calculated. The mean length of stay was also calculated. Descriptive statistics were used to summarise the patients' demographics and hospitalisation cost characteristics. Student's $t$-test was used to examine the difference between the surgical group and the non-surgical group. Linear regression analysis was used to examine the increase in costs due to complications and delays in surgery. Chi-square test or Fisher's exact test was used to examine the difference in the number of comorbidities between the surgical group and non-surgical group. A power calculation showed that the study's power to detect a difference in cost between surgical treatment and non-surgical treatment was above 0.9. All statistical analyses were done using Stata Statistical Software version 10 (StataCorp, College Station, TX, USA).

\section{RESULTS}

During the study period, 272 patients aged $>60$ years were admitted for hip fractures (Fig. 1). Among these patients, 28 were excluded for various reasons (Table I). Thus, a total of 244 patients were included in the study. The mean age of the 244 patients was 80.9 years and 187 (76.6\%) of these patients received surgical treatment.

Patient demographics are presented in Table II. The mean cost of hospitalisation was SGD 13,313.81 and the mean length of stay was 17.13 days. Patient comorbidities are shown in Table III. The number of patients with comorbidities was significantly different when the surgical group was compared with the non-surgical group. In the latter group of patients, there was a significantly higher prevalence of hypertension, ischaemic heart disease and stroke $(p<0.05)$. These patients also had a higher mean age and a higher prevalence of chronic obstructive pulmonary disease that tended toward statistical significance $(p=0.056)$.

The mean cost of hospitalisation was higher for the patients who were treated surgically as compared to those who were treated non-surgically (SGD 14,815.70 vs. SGD 9,011.38, respectively; $p<0.01)$. However, the surgical group had a shorter length of hospital stay than the non-surgical group (15.97 [range 4-56] days vs. 19.25 [range 2-84] days, respectively) (Table II). The mean cost of surgery was SGD 3,952.40 (range SGD 2,551-SGD 5,541) and the mean implant cost was SGD 1,574 (range SGD 386.70-SGD $5,818.90)$. The costs of the surgery and implant(s) contributed to the higher mean cost for the surgical group. The average room and treatment charge per day per patient was SGD 249.10.

If the surgery was delayed (i.e. $>48$ hours after admission), every additional day of delay was found to result in a SGD 575.89 increase in cost. The average cost for the patients whose surgery was delayed was SGD 2,716.63 more than that of the patients who had surgery within 48 hours. Among the 187 patients who

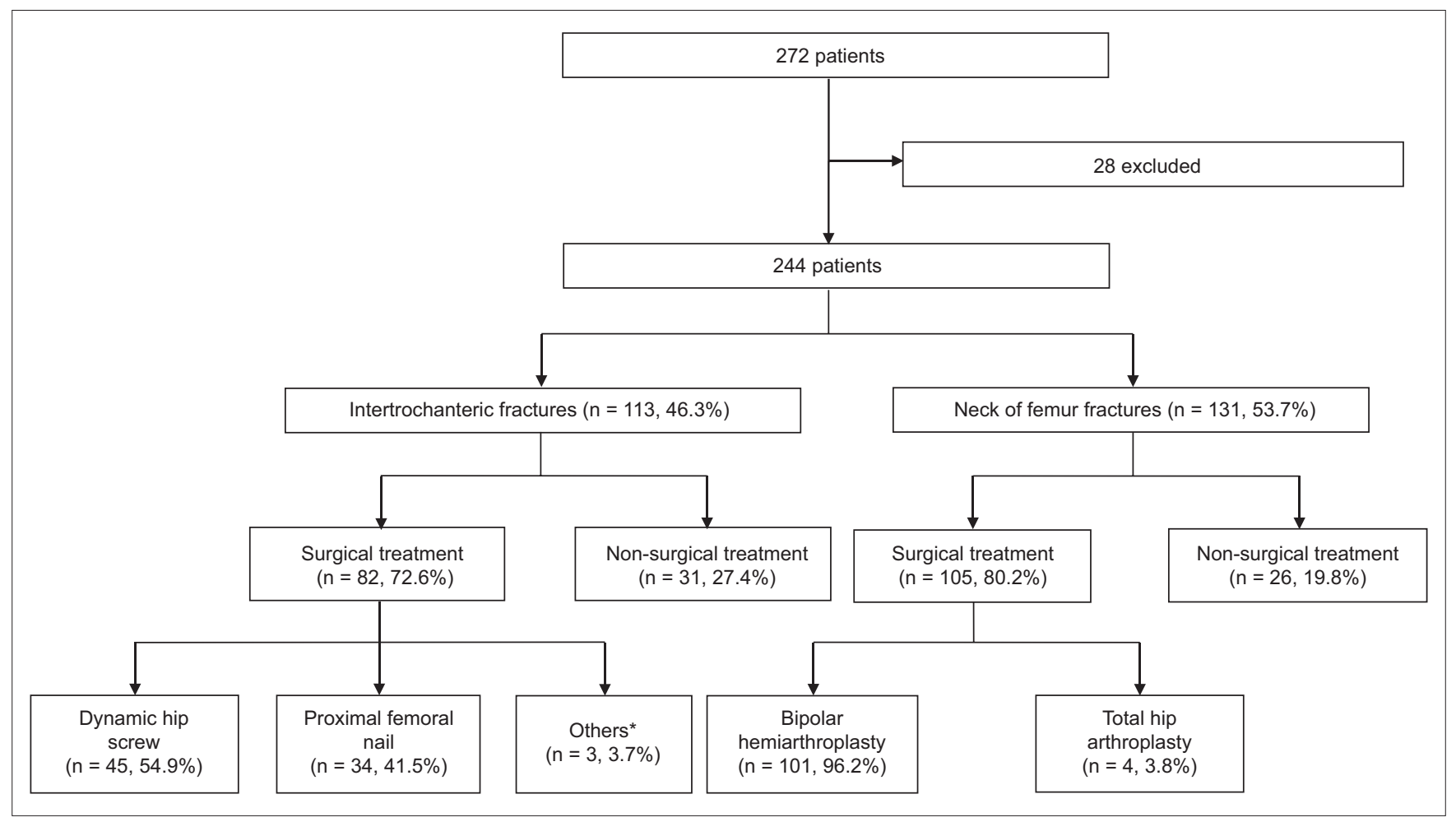

Fig. 1 Flow chart shows patient recruitment and the type of treatment administered. ${ }^{*}$ Others include proximal femoral locking plate $(\mathrm{n}=1)$ and dynamic condylar screw $(n=2)$. 
Table I. Reasons for exclusion ( $n=28$ ).

\begin{tabular}{lc}
\hline Reason & No. \\
\hline Femur shaft fracture & 4 \\
Death before completion of treatment & 9 \\
Discharged to other hospitals during course of treatment & 4 \\
Discharged against medical advice & 1 \\
Complication of previous total hip replacement & 1 \\
Pathological fracture & 1 \\
Periprosthetic fracture & 2 \\
Subtrochanteric fracture & 4 \\
Isolated greater trochanter fracture & 2 \\
\hline
\end{tabular}

Table II. Demographic characteristics of the patients $(n=244)$.

\begin{tabular}{|c|c|c|}
\hline \multirow[t]{2}{*}{ Characteristic } & \multicolumn{2}{|c|}{ No. (\%) } \\
\hline & $\begin{array}{c}\text { Treated } \\
\text { surgically* } \\
\text { (n=187) }\end{array}$ & $\begin{array}{c}\text { Treated } \\
\text { non-surgically } \\
(n=57)\end{array}$ \\
\hline $\mathrm{Age}^{+}(\mathrm{yr})$ & 79.56 & 85.30 \\
\hline \multicolumn{3}{|l|}{ Gender } \\
\hline Male & $46(24.6)$ & $13(22.8)$ \\
\hline Female & $141(75.4)$ & $44(77.2)$ \\
\hline Length of hospital stay ${ }^{+}$(day) & 15.97 & 19.25 \\
\hline Cost $^{+}$(SGD) & $14,815.70$ & $9,011.38$ \\
\hline \multicolumn{3}{|l|}{ Affected hip } \\
\hline Right & $90(48.1)$ & $24(42.1)$ \\
\hline Left & 97 (51.9) & 33 (57.9) \\
\hline
\end{tabular}

*Treated with arthroplasties and/or fixations. +Data presented as mean.

underwent surgical treatment, $78(41.7 \%)$ had the surgery done within 48 hours after admission.

Among the 244 patients, 113 (46.3\%) had intertrochanteric fractures (i.e. AO A1-A3 fractures). Among these 113 patients, 82 underwent surgical treatment and 31 underwent non-surgical treatment. Those that were treated surgically had a lower mean age (79.99 years vs. 85.56 years), lower mean length of hospital stay (15.20 days vs. 21.94 days) and higher mean cost incurred (SGD 13,318.14 vs. SGD 10,457.04). The majority of those who were treated surgically had either a dynamic hip screw (DHS) or proximal femoral nail antirotation (PFNA) implant (Table IV). The average costs of a DHS and PFNA implant were SGD 421.50 and SGD 1,378.50, respectively. The remaining patients who were treated surgically received either a proximal femoral locking plate or dynamic condylar screw implant.

Among the 244 patients, 131 (53.7\%) had neck of femur fractures (i.e. AO B1-B3 fractures). Of these 131 patients, 105 were treated surgically and 26 were treated non-surgically. Total hip arthroplasty was done for four of the 105 patients who were treated surgically; the remainder of the group underwent bipolar hemiarthroplasty. The patients who were treated surgically had a lower mean age (79.23 years vs. 85.02 years) and a higher mean cost incurred (SGD 15,668.22 vs SGD 7,287.80). There was no significant difference in length of hospital stay between the surgical group and the non-surgical group (Table V). The mean implant cost for the bipolar hemiarthroplasties was SGD 2,053.25.
Table III. Patient comorbidities.

\begin{tabular}{lccc}
\hline Comorbidity & \multicolumn{2}{c}{ No. (\%) } & p-value \\
\cline { 2 - 3 } & $\begin{array}{c}\text { Treated } \\
\text { surgically* } \\
\text { (n = 187) }\end{array}$ & $\begin{array}{c}\text { Treated } \\
\text { non-surgically } \\
(\mathbf{n}=\mathbf{5 7})\end{array}$ & \\
\cline { 1 - 3 } Hypertension & $99(52.9)$ & $41(71.9)$ & 0.018 \\
Diabetes mellitus & $63(33.7)$ & $17(29.8)$ & 0.702 \\
Hyperlipidaemia & $74(39.6)$ & $26(45.6)$ & 0.510 \\
Ischaemic heart disease & $18(9.6)$ & $14(24.6)$ & 0.007 \\
Stroke & $26(13.9)$ & $19(33.3)$ & 0.002 \\
Dementia & $24(12.8)$ & $11(19.3)$ & 0.316 \\
Cancer & $15(8.0)$ & $7(12.3)$ & 0.472 \\
Chronic obstructive & $10(5.3)$ & $8(14.0)$ & 0.056 \\
lung disease & $14(7.5)$ & $4(7.0)$ & 1.000 \\
Renal impairment & $9(4.8)$ & $2(3.5)$ & 1.000 \\
Depression & $3(1.6)$ & $1(1.8)$ & 1.000 \\
Schizophrenia & $2(1.1)$ & $0(0)$ & 1.000 \\
Rheumatoid arthritis & & & \\
\hline
\end{tabular}

*Treated with arthroplasties and/or fixations.

Table IV. Characteristics of the patients with intertrochanteric fractures $(n=113)$.

\begin{tabular}{lcc}
\hline Characteristic & \multicolumn{2}{c}{ No. (\%) } \\
\cline { 2 - 3 } & $\begin{array}{c}\text { Treated } \\
\text { surgically* } \\
(\mathbf{n}=\mathbf{8 2})\end{array}$ & $\begin{array}{c}\text { Treated } \\
\text { non-surgically } \\
(\mathbf{n}=\mathbf{3 1})\end{array}$ \\
\hline Gender & $23(28.0)$ & $6(19.4)$ \\
Male & $59(72.0)$ & $25(80.6)$ \\
Female & 79.99 & 85.56 \\
Age ${ }^{+}$(yr) & 15.20 & 21.94 \\
Length of hospital stay $^{+}$(day) & $13,318.14$ & $10,457.04$ \\
Cost $^{+}$(SGD) & & \\
Type of implant $^{\text {DHS }}$ & $45(54.9)$ & NA \\
PFNA & $34(41.5)$ & NA \\
Others & & NA \\
\hline
\end{tabular}

*Treated with fixations. +Data presented as mean. ¥Proximal femoral locking plate or dynamic condylar screw. DHS: dynamic hip screw; NA: not applicable; PFNA: proximal femoral nail antirotation

The list of all the complications encountered among the 244 patients is shown in Table VI. The mean overall cost was SGD 2,689.99 more for the patients who had complications than for those who had no complications $(p=0.011)$.

\section{DISCUSSION}

Hip fracture is a complex and costly medical condition to treat in elderly patients. The elderly are more likely to suffer from osteoporosis and have higher fall risk due to poor balance or the side effects of prescribed medications. Women are more likely to have hip fractures, as they have a higher incidence of osteoporosis. The lifetime risk of hip fractures is $17.5 \%$ for women and $6 \%$ for men. ${ }^{(8)}$ The challenges associated with treating elderly patients are that they are more likely to have multiple comorbidities, be osteoporotic and have problems with post-hospital care. Hip fractures among the elderly also represent a substantial economic 
burden. The Belgian Hip Fracture Study Group demonstrated that there was an increased cost of USD 7,300 per patient per year for elderly hip fracture patients when compared to matched subjects living in the same neighbourhood. ${ }^{(9)}$

In Singapore, the average cost of a hip fracture has risen over the years, from SGD 7,367 and SGD 10,515 in 1993 and 2001, respectively, ${ }^{(6,7)}$ to SGD 13,313 in 2011. As some of this increase in cost may have been a result of inflation, we used the consumer price index to calculate inflation-adjusted costs. We found that even after adjusting for inflation, there was still an increase in the cost of hip fracture over the years (Fig. 2).

In a study by Wong et $\mathrm{al}^{(6)}$ published in 2002, the authors examined patients admitted for hip fractures from 1991 to 1993. The mean hospitalisation time was 17 days and the mean age of the patients was 80.3 years; these results are similar to those of the present study. However, orthogeriatric care had not started at that time and all of the fixations in Wong et al's study were done using dynamic compression screw or cancellous screw. PFNA, a more expensive implant, was not yet in use, which may account for some of the observed difference in overall costs between the two studies. In our study, the mean implant cost was SGD 1,378.50 for PFNA and SGD 421.50 for DHS. A study conducted by Lee et al, ${ }^{(7)}$ published in 2008, collected data on patients admitted for hip fractures in 2001. In their study, the median hospitalisation cost for the patients who were treated surgically was SGD 10,515 and the mean length of hospital stay was 16 days. The median hospitalisation cost for the patients who were treated non-surgically was SGD 16,043 and the mean length of hospital stay was 20 days. The authors also found that a longer delay to surgery and a longer length of hospital stay led to higher costs. ${ }^{(7)}$

In the present study, all patients who were admitted for hip fractures were admitted under the care of an orthopaedic consultant. Those above the age of 60 years were automatically enrolled into the hip fracture care path. This care path takes a multidisciplinary approach and involves several different healthcare professionals (i.e. an orthopaedic surgeon, a geriatric consultant, a case manager, nurses, physiotherapists and occupational therapists). The benefits of such a care path include: ${ }^{(10)}$ (a) a fixed bladder protocol to reduce urinary tract infections; (b) regular analgesia with paracetamol, tramadol and/or mist morphine, as required; (c) chest and limb physiotherapy initiated on admission, and early postoperative mobilisation; (d) fast-track referrals to cardiology for two-dimensional (2D) echocardiography; (e) minimisation of other departmental referrals, as the patient is reviewed by a geriatric consultant; (f) early discharge planning by a case manager; and (g) fixed nursing protocols, including the use of anti-embolic stockings, pneumatic calf pumps and pressure-relieving mattresses, as well as the charting of the patients' input and output, and the monitoring of regular bowel clearance.

Most of the patients in the present study were treated surgically ( $n=187,76.6 \%$ ). These patients had a shorter mean length of hospital stay and higher mean cost incurred as compared to the non-surgical group. The higher cost was due to the costs
Table V. Characteristics of the patients with neck of femur fractures ( $n=131)$.

\begin{tabular}{lcc}
\hline Characteristic & \multicolumn{2}{c}{ No. (\%) } \\
\cline { 2 - 3 } & $\begin{array}{c}\text { Treated } \\
\text { surgically* } \\
(\mathbf{n}=\mathbf{1 0 5})\end{array}$ & $\begin{array}{c}\text { Treated } \\
\text { non-surgically } \\
(\mathbf{n}=\mathbf{2 6})\end{array}$ \\
\hline Gender & & \\
Male & $23(21.9)$ & $7(26.9)$ \\
Female & $82(78.1)$ & $19(73.1)$ \\
Age ${ }^{+}$(yr) & 79.23 & 85.02 \\
Length of hospital stay $^{+}$(day) & 16.57 & 16.04 \\
Cost $^{+}$(SGD) & $15,668.22$ & $7,287.80$ \\
\hline
\end{tabular}

*Treated with arthroplasties. +Data presented as mean.

Table VI. List of complications encountered among the patients ( $n=244)$.

\begin{tabular}{lc}
\hline Complication & No. (\%) \\
\hline Acute myocardial infarction & $2(0.8)$ \\
Acute retention of urine & $2(0.8)$ \\
Atelectasis & $1(0.4)$ \\
Atrial fibrillation & $6(2.5)$ \\
Cement extrusion & $1(0.4)$ \\
Chest infection & $10(4.1)$ \\
Congestive cardiac failure & $1(0.4)$ \\
Deep vein thrombosis & $2(0.8)$ \\
Dengue fever & $1(0.4)$ \\
Haematuria & $1(0.4)$ \\
Hepatobiliary sepsis & $1(0.4)$ \\
Hip dislocation after surgery & $1(0.4)$ \\
Stroke & $1(0.4)$ \\
Thrombocytopenia & $2(0.8)$ \\
Urinary tract infection & $12(4.9)$ \\
\hline
\end{tabular}

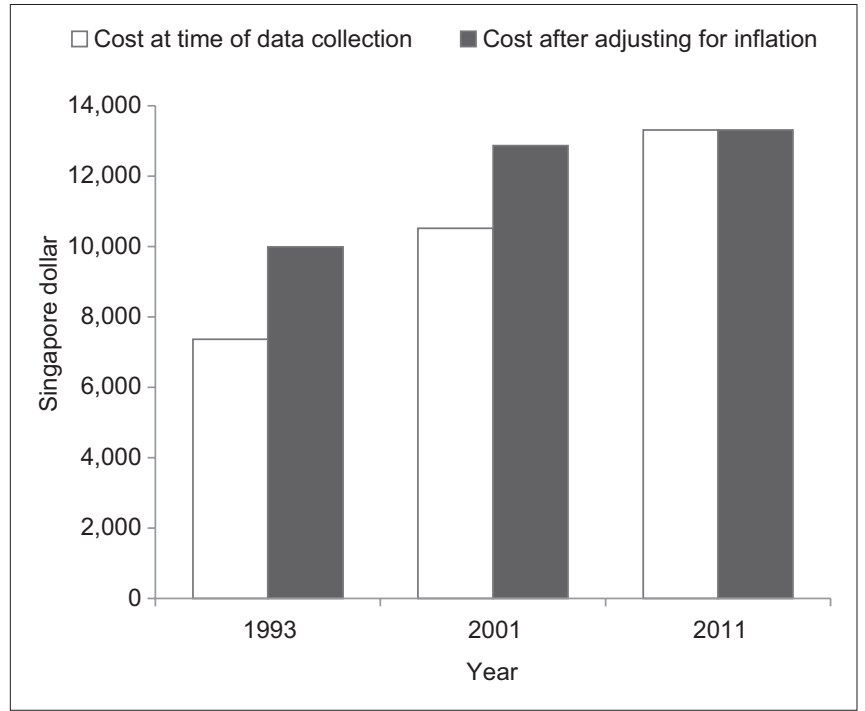

Fig. 2 Cost of hip fracture before and after adjusting for inflation.

of the surgery (mean: SGD 3,952.40) and the implants used (range: SGD 386.70-5,818.90). Among the patients who had intertrochanteric fractures, the patients who were treated nonsurgically had a higher mean age (85.56 years vs. 79.99 years) 
and a significantly longer length of hospital stay (21.94 days vs. 15.20 days) than the patients who were treated surgically; these patients also incurred a lower cost (approximately SGD 2,800 less). Among the patients who had neck of femur fractures, the non-surgical group had a higher mean age than the surgical group (85.02 years vs. 79.23 years); the mean length of stay of the two groups were similar (16.04 days vs. 16.57 days), although there was a great difference in the mean cost incurred by these two groups of patients (approximately SGD 8,380 less in the nonsurgical group).

The mean age of the patients who were treated non-surgically was generally higher than that of the patients who were treated surgically. There was also a higher prevalence of hypertension, ischaemic heart disease, stroke and chronic obstructive pulmonary disease among the patients who were treated nonsurgically. These differences in the age and comorbidity profile of the two groups are expected, as advanced age and the presence of comorbidities are likely to be viewed as deterrents to surgical treatment by the patient and the patient's family.

There has been a shift towards management of elderly hip fractures by both the orthopaedics and geriatrics departments. Della Rocca et $\mathrm{al}^{(4)}$ reviewed two groups of geriatric patients with hip fractures - the first group was managed using historical methods of care, while the second group was treated using a co-management hip fracture protocol (which included the involvement of healthcare staff from internal medicine, anaesthesiology, emergency medicine, rehabilitation, nursing and therapy services). The study found that the co-management group had a lower number of intensive care unit (ICU) admissions, shorter ICU stay, shorter hospitalisation period and lower hospitalisation cost (decreasing from USD 52,323 to USD 38,586).(4)

Although several models for combined care have been published in the literature, no one model has been shown to be superior. Kammerlander et al conducted a literature review comparing different models for combined care and found no best model. ${ }^{(11)}$ They concluded that having integrated care, regardless of the model, can reduce in-hospital mortality, length of hospital stay and time to surgery. ${ }^{(11)}$

Studies have shown that hip fracture patients should be surgically treated as soon as possible, within 48 hours, to reduce mortality and morbidity. ${ }^{(12-14)}$ Khan et al conducted a systemic review of 52 published studies involving 291,413 patients and found that early surgery (defined as within 48 hours) resulted in no adverse outcomes, decreased length of hospital stay and probable reductions in complications and mortality. ${ }^{(12)}$ Other studies have shown that surgery after 48 hours is associated with an increase in patient mortality. ${ }^{(13,14)}$ In the present study, the patients who had surgery after 48 hours had a mean increase in cost of SGD 2,716.63 when compared to those who had surgery within 48 hours. Every additional day of delay to surgery was also found to result in a SGD 575.89 increase in cost, which is higher than the average room and treatment charge per day (SGD 249.10).

Having an integrated care path for patients with hip fractures would help to address these issues. Counselling for surgery starts when patients first arrive in the ward to reduce the time needed to make the decision. The patients are automatically referred to physical therapists, and nurses can start carrying out the standard protocols. Certain investigations can also be fast-tracked; for example, 2D echocardiography can be ordered without having to wait for a consultation with a cardiologist.

Various other centres have demonstrated the cost-effectiveness of having an integrated care system for elderly patients with hip fractures. Kates et al reviewed financial data on older adults who received treatment for hip fractures during a one-year period and demonstrated that the use of an organised programme results in costs that are $66.7 \%$ of the expected costs nationally. ${ }^{(15)}$ Ginsberg et al conducted a cost-utility analysis that integrated the epidemiological and economic aspects of hip fracture patients treated using a comprehensive orthogeriatric model (COGM) of care as compared to patients treated using a standard of care model (SOCM). ${ }^{(16)}$ The authors found that the COGM used 23\% fewer resources per patient than SOCM (\$14,919 vs. \$19,363), demonstrating that the use of an integrated care system for hip fracture patients was cost-effective. ${ }^{(16)}$ In addition, the minimum number of hip fractures per year per centre needs to exceed 72 in order for geriatric hip centres to be economically viable. ${ }^{(17)}$ However, this number is easily achievable in all tertiary hospitals. Below this minimum number, most hospitals will make a loss if they offer geriatric hip fracture care.

In the present study, we only studied the inpatient costs of hip fractures among elderly patients. This does not take into account the cost of any further rehabilitation, if needed, or any additional costs needed for home modifications and subsequent follow-up visits. Further research could be done to determine whether early surgery has any impact on long-term functional outcome and economic burden of hip fractures for both the patient and society. In this study, we were also not able to directly compare the costs of hip fractures in a combined orthogeriatric service and a non-orthogeriatric care centre.

To conclude, the incidence of hip fractures among the elderly will increase with the ageing population, resulting in an increasing economic burden. The current mean cost of hip fractures in the elderly is SGD 13,313.81 per inpatient stay, with the mean cost of surgical treatment being SGD 14,815.70 and the mean cost of non-surgical treatment being SGD 9,011.38. Optimising a patient for surgery as soon as possible will not only reduce mortality and morbidity, but also economic costs. An orthogeriatric comanagement system is economically viable and cost-effective, and is able to improve patient care.

\section{REFERENCES}

1. Gullberg B, Johnell O, Kanis JA. World-wide projections for hip fracture. Osteoporosis Int 1997; 7:407-13.

2. Wiktorowicz ME, Goeree R, Papaioannou A, Adachi JD, Papadimitropoulos E. Economic implications of hip fractures: health service use, institutional care and cost in Canada. Osteoporosis Int 2001; 12:271-8.

3. British Orthopaedic Association, Health Quality Improvement Partnership; The Information Centre. The National Hip Fracture Database National Report 2012. Available at: http://www.nhfd.co.uk/20/hipfractureR.nsf/0/ da44e3a946a14e4180257a6f001eb4db/\$FILE/NHFD\%20National\%20 Report\%202012.pdf. Accessed March 25, 2016. 
4. Della Rocca GJ, Moylan KC, Crist BD, et al. Comanagement of geriatric patients with hip fractures: a retrospective, controlled, cohort study. Geriatr Orthop Surg Rehabil 2013; 4:10-5.

5. Department of Statistics, Singapore. Population Trends 2015. Available at: http://www.singstat.gov.sg/docs/default-source/default-document-library/ publications/publications_and_papers/population_and_population structure/population2015.pdf. Accessed March 25, 2016.

6. Wong MK, Arjandas, Ching LK, Lim SL, Lo NN. Osteoporotic hip fractures in Singapore--costs and patient's outcome. Ann Acad Med Singapore 2002; 31:3-7.

7. Lee YH, Lim YW, Lam KS. Economic cost of osteoporotic hip fractures in Singapore. Singapore Med J 2008; 49:980-4.

8. Melton LJ 3rd. Who has osteoporosis? A conflict between clinical and public health perspectives. J Bone Miner Res 2000; 15:2309-14.

9. Haentjens P, Autier P, Barette M, Boonen S; Belgian Hip Fracture Study Group. The economic cost of hip fractures among elderly women. A one year, prospective, observational cohort study with matched pair analysis. Belgian Hip Fracture Study Group. J Bone Joint Surg Am 2001; 83-A:493-500.

10. Doshi HK, Ramason R, Azellarasi J, Naidu G, Chan WL. Orthogeriatric model for hip fracture patients in Singapore: our early experience and initial outcomes. Arch Orthop Trauma Surg 2014; 134:351-7.

11. Kammerlander C, Roth T, Friedman SM, et al. Ortho-geriatric service--a literature review comparing different models. Osteoporosis Int 2010; 21(Suppl 4):S637-46.

12. Khan SK, Kalra S, Khanna A, Thiruvengada MM, Parker MJ. Timing of surgery for hip fractures: a systemic review of 52 published studies involving 291,413 patients. Injury 2009; 40:692-7.

13. Trpeski S, Kaftandziev I, Kjaev A. The effects of time-to-surgery on mortality in elderly patients following hip fractures. Prilozi 2013; 34:116-21.

14. Dailiana Z, Papakostidou I, Varitimidis S, et al. Surgical treatment for hip fractures: factors affecting mortality. Hippokratia 2013; 17:252-7.

15. Kates SL, Mendelson DA, Friedman SM. The value of an organized fracture program for the elderly: early results. J Orthop Trauma 2011; 25:233-7.

16. Ginsberg G, Adunsky A, Rasooly I. A cost-utility analysis of a comprehensive orthogeriatric care for hip fractures patients, compared with standard of care treatment. Hip Int 2013; 23:570-5.

17. Clement RC, Ahn J, Mehta S, Bernstein J. Economic viability of geriatric hip fracture centers. Orthopedics 2013; 36:e1509-14. 